\title{
High Return Performance of Calcium Phosphonate (Ca-DTPMP) Colloidal Inhibitors for Squeeze Treatment in Shale Formations
}

\author{
Shujun Gao*, Chong Dai, Xin Wang, Yue Zhao, Yi-Tsung Lu, Guannan Deng, \\ Saebom Ko, Samiridhdi Paudyal, Khadouja Harouaka, Amy T. Kan and Mason Tomson \\ Department of Civil and Environmental Engineering, Rice University, Houston, TX, United States
}

OPEN ACCESS

Edited by:

Qing You,

China University of Geosciences,

China

Reviewed by:

Hock Jin Quah,

University of Science Malaysia,

Malaysia

Tongtao Yue,

China University of Petroleum, China Houjian Gong,

China University of Petroleum, China

${ }^{*}$ Correspondence:

Shujun Gao

shujun.gao@bakerhughes.com

Specialty section:

This article was submitted to Colloidal Materials and Interfaces, a section of the journal

Frontiers in Materials

Received: 10 March 2020

Accepted: 28 July 2020

Published: 31 August 2020

Citation:

Gao S, Dai C, Wang $X$, Zhao $Y$,

Lu Y-T, Deng G, Ko S, Paudyal S, Harouaka K, Kan AT and Tomson M

(2020) High Return Performance

of Calcium Phosphonate (Ca-DTPMP)

Colloidal Inhibitors for Squeeze

Treatment in Shale Formations.

Front. Mater. 7:285

doi: 10.3389/fmats.2020.00285
The objective of this work was to develop nanomaterial inhibitor with long squeeze life for oil and gas production. Different surfactants, such as trisodium citrate ( $\left.\mathrm{Na}_{3} \mathrm{Cit}\right)$, cetyltrimethyl ammonium bromide, benzethonium chloride (Hyamine), and bipyridinium dibromide (Gemini), were added to synthesize nanoparticle Ca-DTPMP inhibitor at pH 9.0. The inhibitors were characterized by scanning electron microscope and dynamic light scattering. All these surfactants significantly decreased the particle size, but Gemini was the most effective one to control the calcium phosphonate salt particle size to be about $500 \mathrm{~nm}$. Long-term squeeze experiments ( $3000 \mathrm{PV}$ ) saturated with calcite at $70^{\circ} \mathrm{C}$ and 75 psi back pressure through a glass column packed with Fayetteville shale rock show that the return DTPMP concentration was as high as 3 ppm for the GeminiCa-DTPMP colloidal inhibitor. This is an unprecedented improvement in comparison with the squeeze return of DTPMP only inhibitor where the inhibitor return concentration dropped to below 1 ppm within 400 pore volumes. The Ca-DTPMP speciation, potential field application, and the SqueezeSoftPitzer model prediction are also discussed.

Keywords: Ca-DTPMP, nanoparticle, scale inhibitor, squeeze treatment, shale formation

\section{INTRODUCTION}

Scale formation has been a continuous challenge associated with the oil and gas industry. Scale formation can cause severe blockage problems in downhole equipment, wellbore, production tubulars, pump, separator, and so on, resulting in production decline, formation damage, increased cost, and even well shut-in (Guo et al., 2012; Sutherland et al., 2013; Veisi et al., 2018).

Squeeze treatment is one of the most effective and long-lasting approaches for scale inhibition and has been widely applied in the oilfield (Fleming et al., 2010; Zhang et al., 2011). In a squeeze treatment, scale inhibitor is injected (pill injection) into the well and retained on the formation rock by precipitation and/or adsorption during the well shut-in period. Once the well starts production, the inhibitor will gradually desorb, release, and flow back with the production water. If the inhibitor return concentration is higher than the required minimum inhibitor concentration (MIC), the scale 
formation will be inhibited (Vazquez et al., 2012). Therefore, one significant objective in squeeze research is to extend the squeeze lifetime, i.e., to maintain the return concentration above MIC as long as possible. Several methods have been attempted including (1) enhancers addition in preflush (Ghosh and Li, 2013; Sutherland and Jordan, 2016) or pill injection (Jordan et al., 2001; Selle et al., 2003), such as divalent metal ion $\mathrm{Fe}^{2+}$ and polyaspartic acid; (2) newly developed inhibitors such as $\gamma$ $\mathrm{AlO}(\mathrm{OH})$ nanoparticle in which the squeeze life time improved 60 times (Poynton et al., 2004; Vazquez et al., 2006; Yan C. et al., 2014); and (3) optimization of modeling design (Mackay and Jordan, 2003; Kan et al., 2005) such as SQUEEZE V and SqueezeSoftPitzer.

Previous results have shown that $\mathrm{Ca}^{2+}$ ion and DTPMP (diethylenetriamine pentamethylene phosphonic acid) can precipitate (denoted as Ca-DTPMP) and form a slurry solution. This Ca-DTPMP slurry inhibitor has a much longer squeeze life than the normal liquid DTPMP inhibitor (Ruan et al., 2016). The later DTPMP return concentration is below $1 \mathrm{ppm}$ after 400 pore volumes (PV) flow back tested in Ottawa sand, while that is still above 1 ppm after 1500 PV using Ca-DTPMP inhibitor (Ruan et al., 2016). This was attributed to the large surface area to volume ratio and adsorption rate of the Ca-DTPMP nanomaterial inhibitor; the release kinetics was therefore favored. The particles were well developed, in other words, aged by successive dissolution to remove the high solubility phosphonate materials before use in the squeeze. It could gradually release inhibitor (long-term flowback). However, under certain conditions, $1 \mathrm{ppm}$ is still not high enough especially at higher temperature conditions and brine higher saturation index values (SI) (Zhang et al., 2014). To better control the solubility and dissolution thermodynamics of the Ca-DTPMP inhibitor, one of the simplest and cost-effective approaches is to add different surfactants. Surfactants can greatly reduce surface tension and inhibit aggregation by providing electrostatic double-layer repulsions. Surfactants can also self-assemble to micelles and supply a soft template for formation of different nanostructures (Zargartalebi et al., 2015; Kamal, 2016).

Shale reservoirs have grown rapidly in recent years (Horner et al., 2016; Kilian, 2016). Fracking is frequently required in shale oil production. Unlike conventional carbonate formations, shale consists of not only carbonate but also large amount of quartz, clay, and some minor minerals. However, limited squeeze research has been conducted in shale formation (Yan et al., 2015). Whether the colloidal CaDTPMP inhibitor can be applied in shale oil well and the return behavior remain unknown.

In this study, different frequently used surfactants were used to synthesize Ca-DTPMP nanomaterial inhibitors. The particle morphologies were characterized by SEM and transmission electron microscopy (TEM). Longterm (up to $3000 \mathrm{PV}$ ) squeeze tests were carried out in glass column packed with Fayetteville shale. The work shows very promising potential application to squeeze treatment for the oil and gas industry using Ca-DTPMP nanomaterial inhibitors.

\section{EXPERIMENTAL PROCEDURE}

Ca-DTPMP particles were prepared by following the same procedure described previously (Ruan et al., 2016). First, $1.10 \mathrm{~g}$ $(0.25 \mathrm{M})$ calcium chloride dihydrate $\left(\mathrm{CaCl}_{2} \cdot 2 \mathrm{H}_{2} \mathrm{O}\right)$ was added into $30 \mathrm{ml}$ deionized water $\mathrm{H}_{2} \mathrm{O}$. Four surfactants, trisodium citrate $\left(\mathrm{Na}_{3} \mathrm{Cit}\right)$, cetyltrimethyl ammonium bromide (CTAB), benzethonium chloride (Hyamine), and bipyridinium dibromide (Gemini), were then added in each separate experiments, respectively. Their formula and structures are listed in Table $\mathbf{1 .}$ The molar concentration for all surfactants was kept at 0.05 $\mathrm{M}$ (mass per liter: $0.44 \mathrm{~g} \mathrm{Na}{ }_{3} \mathrm{Cit} \cdot 2 \mathrm{H}_{2} \mathrm{O}, 0.55 \mathrm{~g} \mathrm{CTAB}, 0.67 \mathrm{~g}$ Hyamine, or $0.81 \mathrm{~g}$ Gemini). The solution was stirred until clear which typically took $10 \mathrm{~min}$. The next step was to add $6.75 \mathrm{~g}$ of 15.9 wt.\% DTPMP solution dropwise (Ca/DTPMP $=4)$ to each of the aforementioned solutions. White milky Ca-DTPMP precipitates were observed immediately. The $\mathrm{pH}$ was adjusted to

TABLE 1 | Formula and structures of different surfactants used in this study.

\begin{tabular}{lll}
\hline Name Abbreviation Formula & Structure (Takeoka et al., 2005; \\
& Sydam et al., 2013; Thottoli and \\
& Unni, 2013; Tsagkaropoulou et al., \\
& \\
&
\end{tabular}

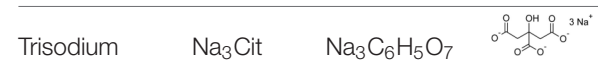

citrate

Cetyltrimethyl CTAB $\mathrm{C}_{16} \mathrm{H}_{33} \mathrm{~N}\left(\mathrm{CH}_{3}\right)_{3} \mathrm{Br}$

ammonium

bromide

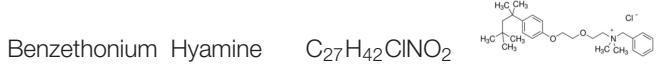

chloride

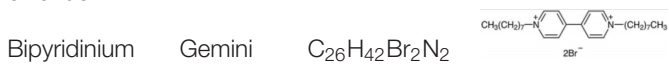

dibromide

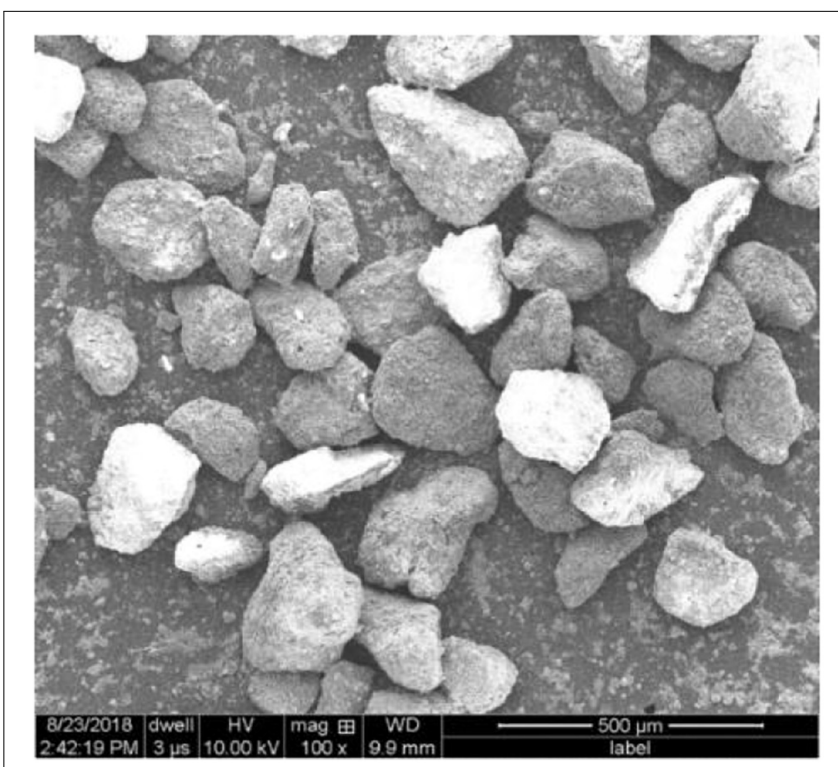

FIGURE 1 | SEM image of Fayetteville shale rocks (106-250 $\mu \mathrm{m})$. 

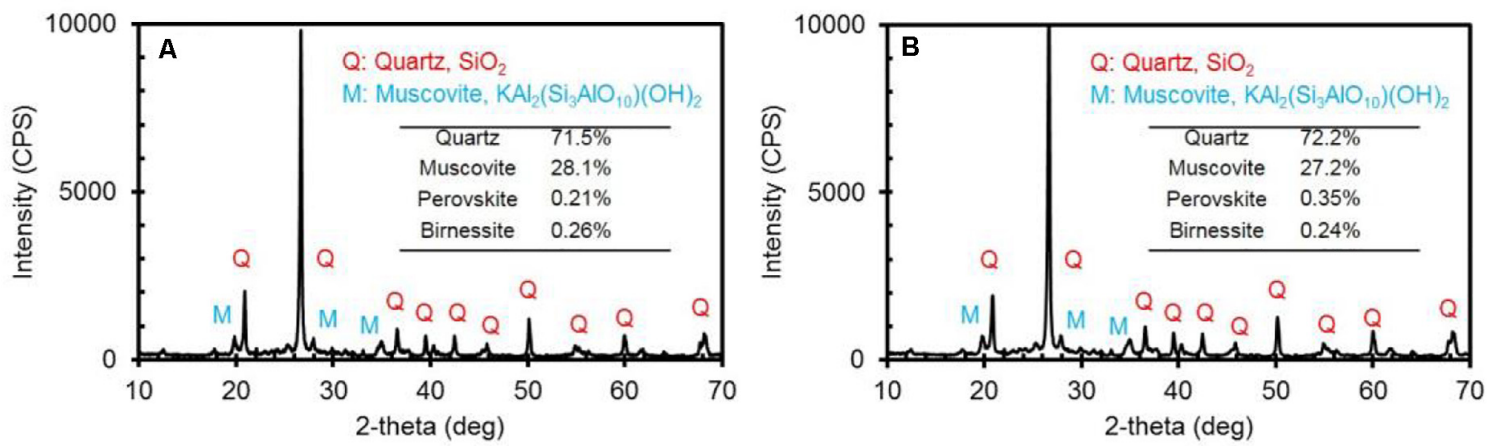

FIGURE 2 |XRD patterns of Fayetteville shale: (A) 106-250 $\mu \mathrm{m}$, washed by 1\% acetic acid; (B) <106 $\mu \mathrm{m}$, no wash.

9.0 (Franco-Aguirre et al., 2018) with $1 \mathrm{M} \mathrm{NaOH}$. The solution was then placed in a water bath at $90^{\circ} \mathrm{C}$ for $24 \mathrm{~h}$. Finally, the solution was centrifuged at $4000 \mathrm{rpm}(\mathrm{g}$ factor $=1968)$ for $4 \mathrm{~min}$. The supernate was poured out and disregarded. DI $\mathrm{H}_{2} \mathrm{O}$ was added to the solid, then stirred, centrifuged, and disregarded for three times. The final solid was topped to $15 \mathrm{ml}$ with $\mathrm{DI} \mathrm{H}_{2} \mathrm{O}$. ICP measurement shows the $\mathrm{Ca}^{2+}$ and DTPMP concentration were 0.21 and $0.74 \mathrm{~g} / \mathrm{L}$, respectively, giving the stoichiometry of the final colloidal slurry close to $\mathrm{Ca}_{4} \mathrm{H}_{2} \mathrm{DTPMP}$, which is similar to previous results (Zhang et al., 2017). According to mass balance calculation, $70 \%$ yield was achieved using this preparation method.

SEM images were obtained from FEI Quanta 499 ESEM FEG at $15 \mathrm{keV}$ for morphology characterization. X-ray diffraction (XRD) patterns were measured from a Rigaku D/max Ultra II Powder Differactometer equipped with $\mathrm{Cu} \mathrm{K \alpha}$ radiation source at $40 \mathrm{kV}$ and $40 \mathrm{~mA}$. TEM image was recorded from JEOL 2010 transmission electron microscope at an acceleration voltage of $100 \mathrm{kV}$. The prepared sample was dispersed in deionized water and then deposited on a copper grid with carbon film followed by slow solvent evaporation. The particle size distribution was measured by dynamic light scattering (DLS; Brookhaven Instruments 90Plus). Samples were diluted 30 times to avoid aggregation before the measurement.

Fayetteville shale was ground and sieved to $106-250 \mu \mathrm{m}$, then washed by $1 \%$ acetic acid and DI water to remove fines and highenergy particle edges, and finally dried in the oven. Figure $\mathbf{1}$ shows the morphology of the particles. The XRD patterns shown in Figure $2 \mathrm{~A}$ indicate that quartz $\left(\mathrm{SiO}_{2}, 71.5 \%\right)$ is the main component, followed by Muscovite $\left(\mathrm{KAl}_{2}\left(\mathrm{Si}_{3} \mathrm{AlO}_{10}\right)(\mathrm{OH})_{2}\right.$, $28.1 \%$ ), and some other minor impurities such as Perovskite and Birnessite. It is necessary to point out that the sieving or washing process had no obvious effect on the shale rock composition. As demonstrated in Figure 2B, the XRD quantitative analysis shows that the composition is almost the same for the rock particles size below $106 \mu \mathrm{m}$ and without washing.

The 106-250 $\mu \mathrm{m}$ particles were tightly packed into a glass column (ID $6.6 \mathrm{~mm} \times \mathrm{L} 10 \mathrm{~mm}$ ). The total packed shale solid mass was $\sim 1.28 \mathrm{~g}$ with a porosity of 0.48 , giving a pore volume (PV) of approximately $1 \mathrm{ml}$. However, the frits were not placed during pill treatment to inject the colloidal inhibitor smoothly. Squeeze experiment was carried out at $70^{\circ} \mathrm{C}$ in a water bath in the following steps:

1. Pre-flush: flush the packed column with $10 \mathrm{ml}$ of $1 \mathrm{M} \mathrm{NaCl}$, $0.1 \mathrm{M} \mathrm{CaCl}_{2}, \mathrm{pH}$ 6.7, 5 mM PIPES (piperazine-N,N'-bis(2ethanesulfonic acid). A 75-psi backpressure check valve was connected before the effluent;

2. Pill injection: inject $0.9 \mathrm{PV}$ of the as-prepared Ca-DTPMP inhibitor (4 wt.\% as DTPMP). It is worth noting that this laboratory squeeze experiment is called "totally contained squeeze" (Kan et al., 2004) to simulate all of the injected inhibitor contained in the column;

3. Over-flush: flush $0.1 \mathrm{PV}$ of the same solution as in pre-flush to push the inhibitor more forward. Two $10 \mu \mathrm{m}$ PTFE frits were placed at both ends of the column after over-flush;

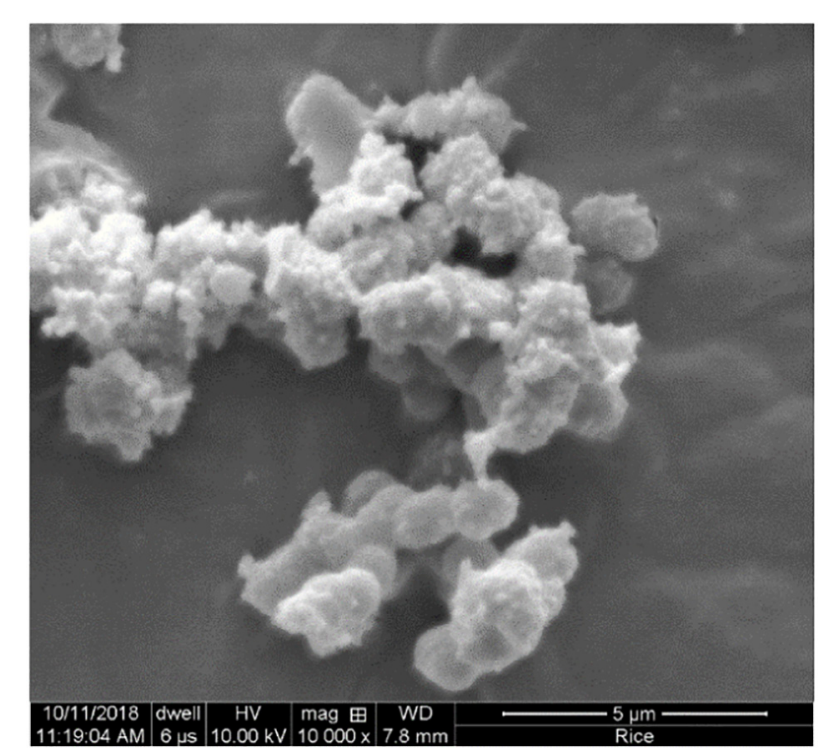

FIGURE 3 | SEM image of Ca-DTPMP particles prepared without adding surfactant. 

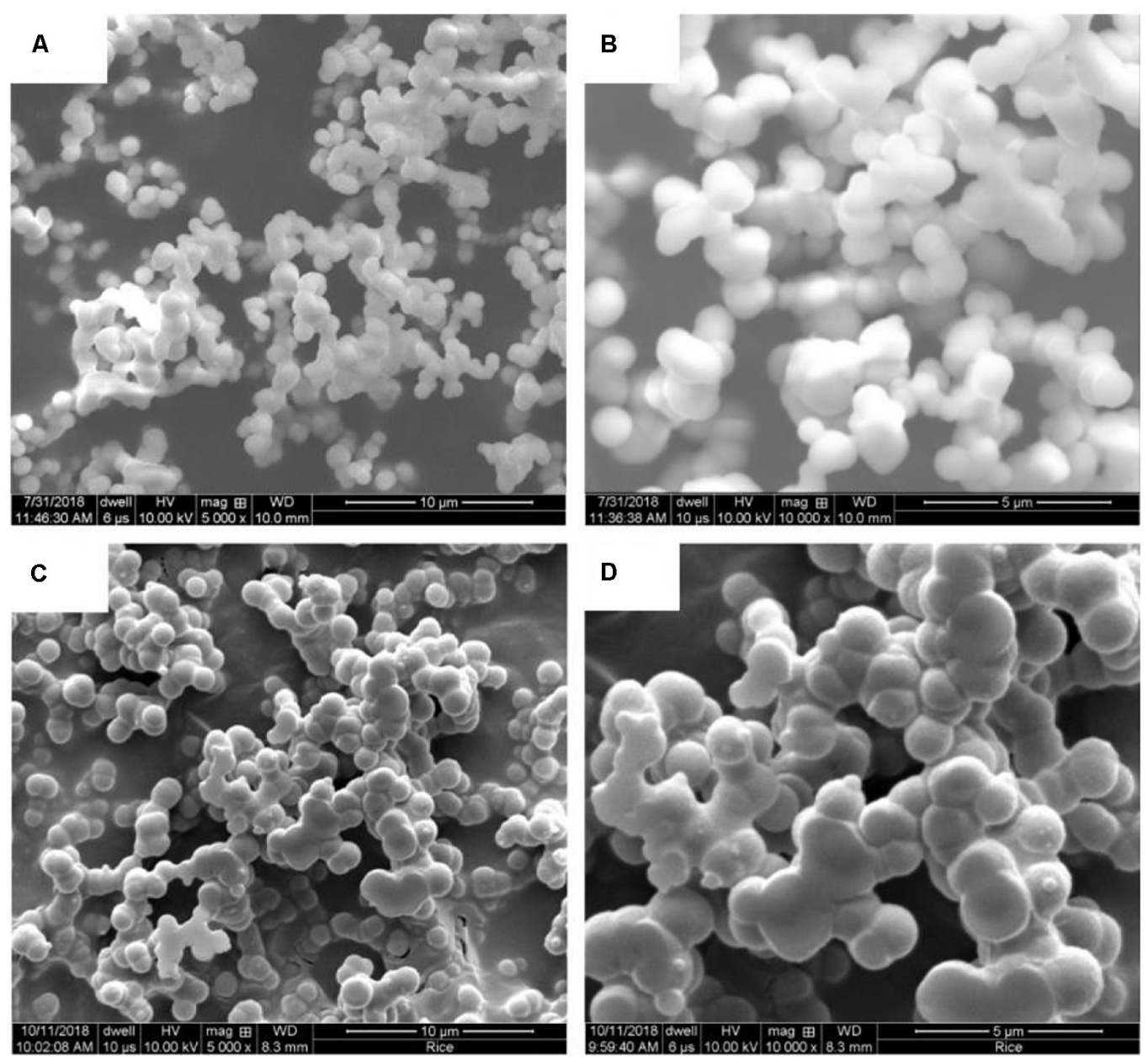

FIGURE 4 | SEM images of Ca-DTPMP particles prepared in the presence of (A,B) $\mathrm{Na}_{3}$ Cit and (C,D) CTAB.

4. Shut-in: close both the ends and place the column in the water bath for $24 \mathrm{~h}$ to let the inhibitor interact with the formation rock;

5. Flow back: the production brine was selected as $1 \mathrm{M}$ $\mathrm{NaCl}, 0.025 \mathrm{M} \mathrm{CaCl}_{2}, 0.015 \mathrm{M} \mathrm{NaHCO}_{3}$, pH 5.6, purged with 1 bar $\mathrm{CO}_{2}$ gas at $70^{\circ} \mathrm{C}$. Under this condition, $\mathrm{Ca}^{2+}$ and $\mathrm{CO}_{3}{ }^{2-}$ concentrations were in equilibrium $(\mathrm{SI}=0)$ with respect to calcite. The flow rate was maintained at $8 \mathrm{ml} / \mathrm{h}$, corresponding to a linear production rate of $37.6 \mathrm{ft} /$ day, in the reverse direction as the pill injection. The effluent was collected by a fraction collector and analyzed by inductively coupled plasma optical emission spectrometry (ICP-OES, Optima 4300Dv; Perkin Elmer 2011) for phosphorus concentration.

\section{EXPERIMENTAL RESULTS}

\section{Ca-DTPMP Characterization}

Figures 3-5 show the Ca-DTPMP particles prepared with and without surfactant. The diameter of Ca-DTPMP particle without surfactant (Figure 3) is close to $3 \mu \mathrm{m}$, which is confirmed with DLS measurements, shown in Figure 6. Attempts were made to inject these large crystalline Ca-DTPMP particles into the packed column; however, injection failed as a result of too much pressure building up.

Surfactants were added to reduce the particle size and to better control the dissolution behavior. The morphologies of Ca-DTPMP particles prepared in the presence of $\mathrm{Na}_{3} \mathrm{Cit}$, $\mathrm{CTAB}$, Hyamine, and Gemini are shown in Figures 4, 5, respectively. After adding surfactant, all the particle sizes decreased, compared with the particles without surfactant addition shown in Figure 3. For $\mathrm{Na}_{3} \mathrm{Cit}$ and CTAB, the particle sizes are less than $1 \mu \mathrm{m}$. Hyamine and Gemini surfactants showed even better performance and further decreased particle size to $\sim 500 \mathrm{~nm}$. Figure 6 shows the DLS measurements and the average particle sizes are summarized in Table 2. Gemini surfactant shows the best performance as it has two hydrophilic heads and two hydrophobic tails. It can easily form micelles or vesicles and restrict the Ca-DTPMP crystal growth. In addition, the particle morphology also changed from irregular shape to spherical and became more uniform 

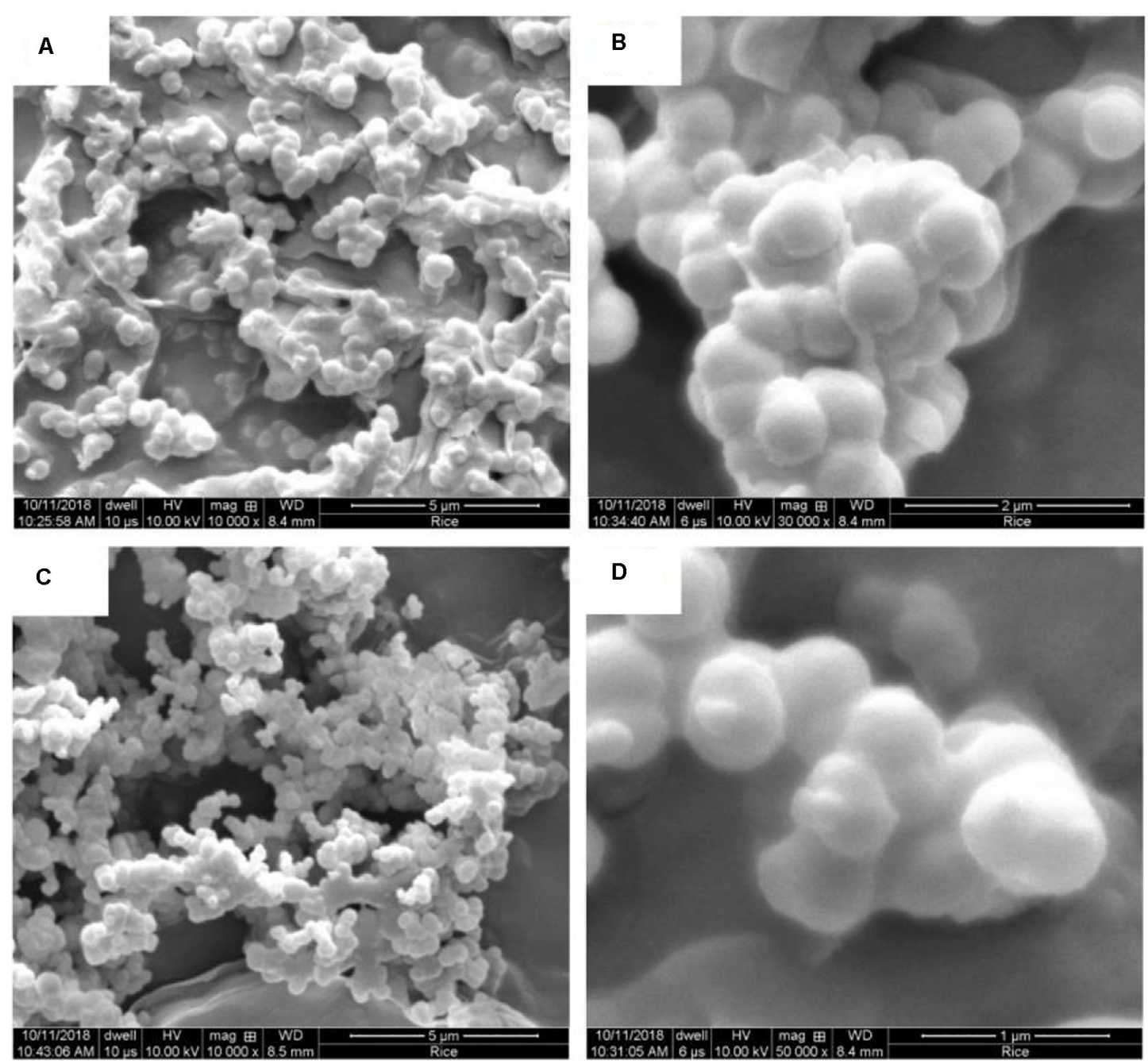

FIGURE 5 | SEM images of Ca-DTPMP particles prepared in the presence of $\mathbf{( A , B ) ~ H y a m i n e ~ a n d ~ ( C , D ) ~ G e m i n i . ~}$

after surfactant addition. A TEM characterization of CaDTPMP prepared with Gemini is shown in Figure 7. The stability and compatibility of the Ca-DTPMP inhibitor in high temperature and high salinity brine may be required in the future work.

\section{Squeeze Return Behavior}

Long-term squeeze tests were performed to evaluate the return behavior of Ca-DTPMP inhibitors synthesized with different surfactants. For comparison, liquid DTPMP only inhibitor ( $\mathrm{pH} \sim 6.6$ ) was tested first, as shown in Figure 8. All the initial return values are between 100 and $1000 \mathrm{ppm}$, then drop to several ppm after a few hundred PVs. At the conditions of these experiments, DTPMP only inhibitor shows poor retention; the concentration quickly decreased below $1 \mathrm{ppm}$, before $400 \mathrm{PV}$. This is in contrast with common experience that DTPMP generally squeezes well. The possible reason will be discussed later. While Ca-DTPMP inhibitor prepared in the presence of $\mathrm{Na}_{3} \mathrm{Cit}$ could still remain above 1 ppm for $2000 \mathrm{PVs}$, return performance was almost five times longer compared with DTPMP only inhibitor. With CTAB and Hyamine surfactants, the DTPMP return concentrations were further improved to $\sim 2 \mathrm{ppm}$ up to $3000 \mathrm{PV}$. In the return curve with Gemini surfactant, the DTPMP concentration is as high as 3 ppm even after 3000 PV. This is an unprecedented improvement compared with a previous study (Ruan et al., 2016). Comparing with other nanoparticle inhibitor studies, the results also show better performance. For example, Guraieb et al. (2019) developed a nanoparticle inhibitor and the squeeze return concentration decreased to below 1 ppm after 400 PV. Yan C. et al. (2014) found that $\gamma$ - $\mathrm{AlO}(\mathrm{OH})$ nanoparticle could also improve the squeeze performance, but the concentration trended less than 1 ppm after 1000 PV.

DTPMP mass balance with PV was calculated to better understand the inhibitor retention behavior on the shale rock, as shown in Figure 9. The total mass return of the first $10 \mathrm{PV}$ is defined as the initial loss. For DTPMP 




Particle size, $\mathrm{nm}$

FIGURE 6 | DLS measurement of Ca-DTPMP particles prepared with and without surface surfactant.

TABLE 2 | Average particle diameter sizes from DLS measurements.

\begin{tabular}{lc}
\hline Surfactant & Ca-DTPMP average size, $\mathbf{n m}$ \\
\hline No surfactant & 3025 \\
$\mathrm{Na}_{3} \mathrm{Cit}$ & 934 \\
$\mathrm{CTAB}$ & 855 \\
Hyamine & 732 \\
Gemini & 536
\end{tabular}

only inhibitor, the initial loss is above 60\%, i.e., a large fraction of the inhibitor was non-effective, whereas for $\mathrm{Ca}$ DTPMP inhibitor prepared with surfactants, the initial loss is less than $20 \%$ and gradually increased to $60 \%$ after 3000 PV. These results demonstrate that liquid DTPMP inhibitor has a poor attachment onto the shale formation and a large amount of the mass flowed out in the initial few PVs, whereas Ca-DTPMP nanomaterial was well attached and efficiently retained onto the shale formation in the presence of surfactant.

The Ca-DTPMP speciation has been established by Tomson et al. (1994). The logarithm of ion activity product [ $\log ($ IP)] changes during a squeeze test and may be an indication of saturation or phase change. In this study, the DTPMP ion activity is assumed to be the same as the return concentration measured in Figure 8, and $\mathrm{Ca}^{2+}$ is $1000 \mathrm{ppm}$. Figure 10 shows the $\log (\mathrm{IP})$ value with number of PV. Basically, the trend is the same as observed in Figure 8 because at a constant $\mathrm{Ca}^{2+}$ and $\mathrm{pH}$, only total dissolved DTPMP varies among the ion products. $\mathrm{p} K_{\mathrm{sp}, 1}$ (50.5), $\mathrm{p} K_{\mathrm{sp}, 2}$ (52.9), and $\mathrm{p} K_{\mathrm{sp}, 3}$ (54.0) correspond to amorphous, middle, and crystalline phase of $\mathrm{Ca}_{4} \mathrm{H}_{2}$ DTPMP, respectively (Zhang et al., 2016b). Amorphous phase has the highest solubility. For DTPMP only inhibitor, pIP values quickly decrease toward crystalline phase which has a much lower solubility than the middle phase. For Ca-DTPMP inhibitors prepared with surfactants,

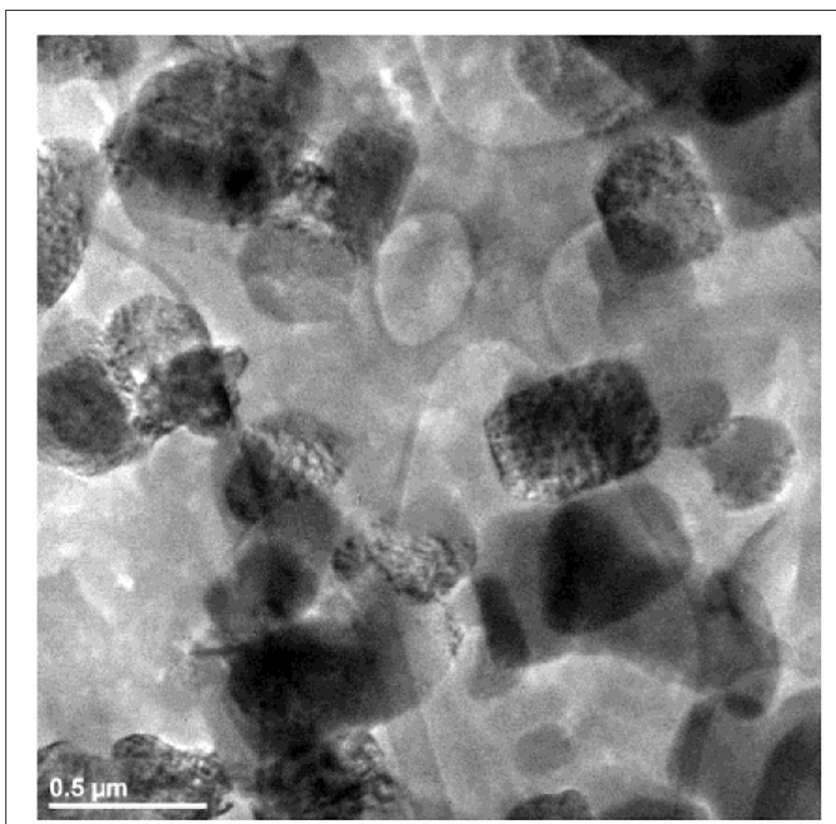

FIGURE 7 | TEM image of Ca-DTPMP inhibitor prepared in the presence of Gemini surfactant.

the pIP also decreases but in a relatively slower manner, then stays around $\mathrm{p} K_{\mathrm{sp}, 2}$, of the middle phase, up to 3000 PV. This agreement with the middle $K_{\text {sp, } 2}$ suggests that the process, as measured, is probably controlled by a thermodynamic process, instead of e.g., desorption kinetics. The surfactants have kinetically prevented its transition from the middle phase to the lower solubility crystalline phase, and consequently the return could be maintained at a higher concentration. 


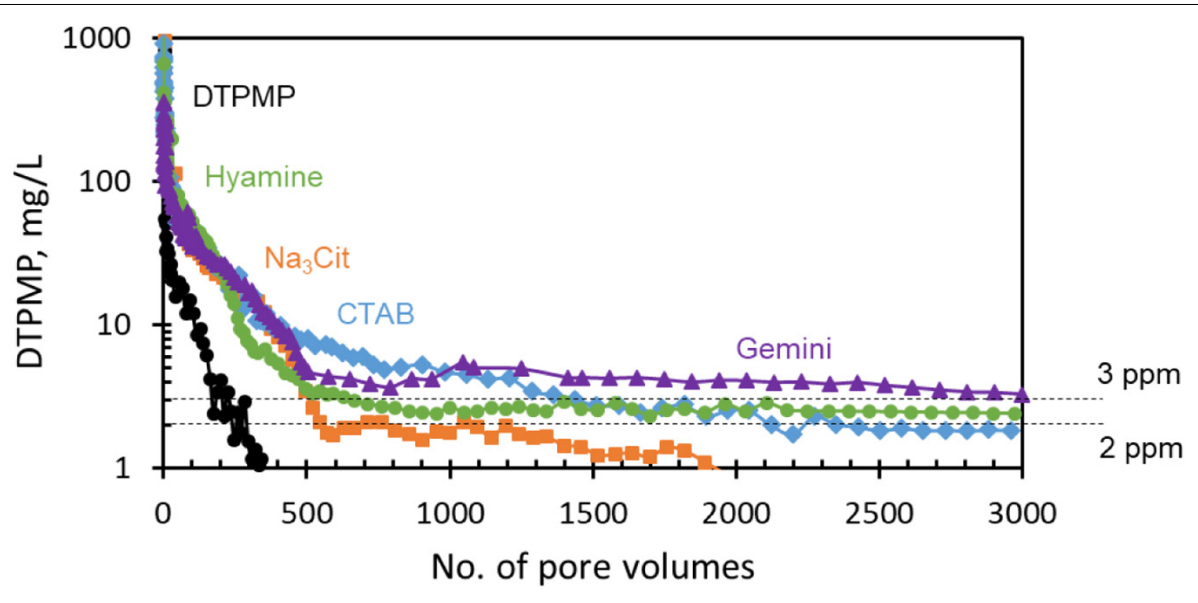

FIGURE 8 | DTPMP return concentrations in laboratory column squeeze test.

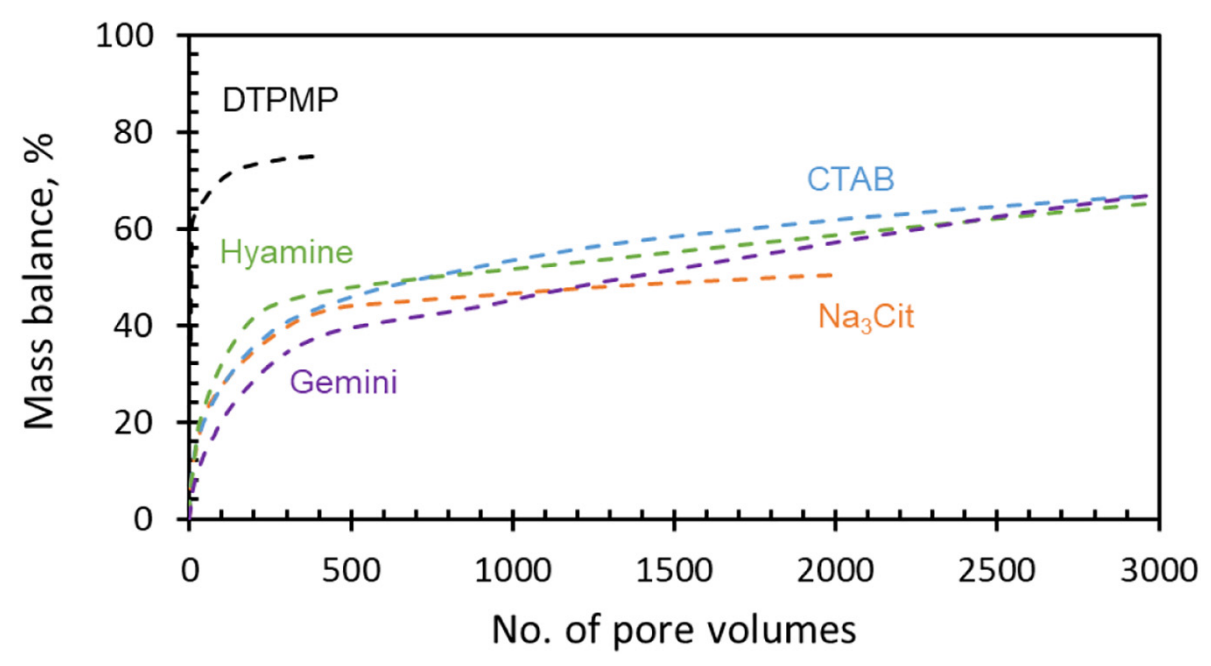

FIGURE 9 | Calculated DTPMP mass balance with PV.

It has been reported that the main interaction mechanism between scale inhibitor and carbonate formation is the precipitation of calcium and inhibitor, whereas in sandstone formation, adsorption is suggested to be the primary mechanism (Yan et al., 2015). Shale formation contains a mixture of quartz, clay, and carbonate minerals. Therefore, the interaction mechanism between Ca-DTPMP inhibitors and shale formations could be both precipitation and adsorption.

After squeeze tests, the shale at different locations in the column was taken out and dissolved in $2 \% \mathrm{HNO}_{3}$ to analyze the remaining DTPMP amount, as shown in Figure 11. In the figure, the inhibitor injection direction was from right to left, whereas the squeeze flow back was in the opposite direction. The liquid DTPMP only shows a uniform distribution though the shale formation, whereas for all the Ca-DTPMP colloidal inhibitors, there is accumulation in the injection end, especially when the particle is larger such as in the presence of $\mathrm{Na}_{3} \mathrm{Cit}$ and CTAB surfactants. More work is required to further reduce the particle size to avoid possible pressure accumulation (Mahmoud, 2014).

\section{Potential Field Application}

The efficiency and activity of the returned DTPMP inhibitor was evaluated using the laser turbidity test described previously (Dai et al., 2019). Ca-DTPMP inhibitor prepared with Gemini returned after squeeze test was selected to test its inhibition to barite $\left(\mathrm{BaSO}_{4}\right)$ scale, as shown in Figure 12. The induction time (when the scale starts to form) without inhibitor is only a few seconds. When adding $1 \mathrm{ppm}$ inhibitor, the induction time increased to $\sim 800 \mathrm{~s}$. More than 2 -h induction time was observed using $3.4 \mathrm{ppm}$ inhibitor, which is in good agreement with the 2-h protection model ScaleSoftPizer (SSP 2019) (Dai et al., 2019). Therefore, the returned Ca-DTPMP inhibitor is still efficient and active. According to the SSP 2019 calculation, 3 ppm DTPMP can inhibit $\mathrm{CaCO}_{3}$ scale formation with $\mathrm{SI}$ as high as 


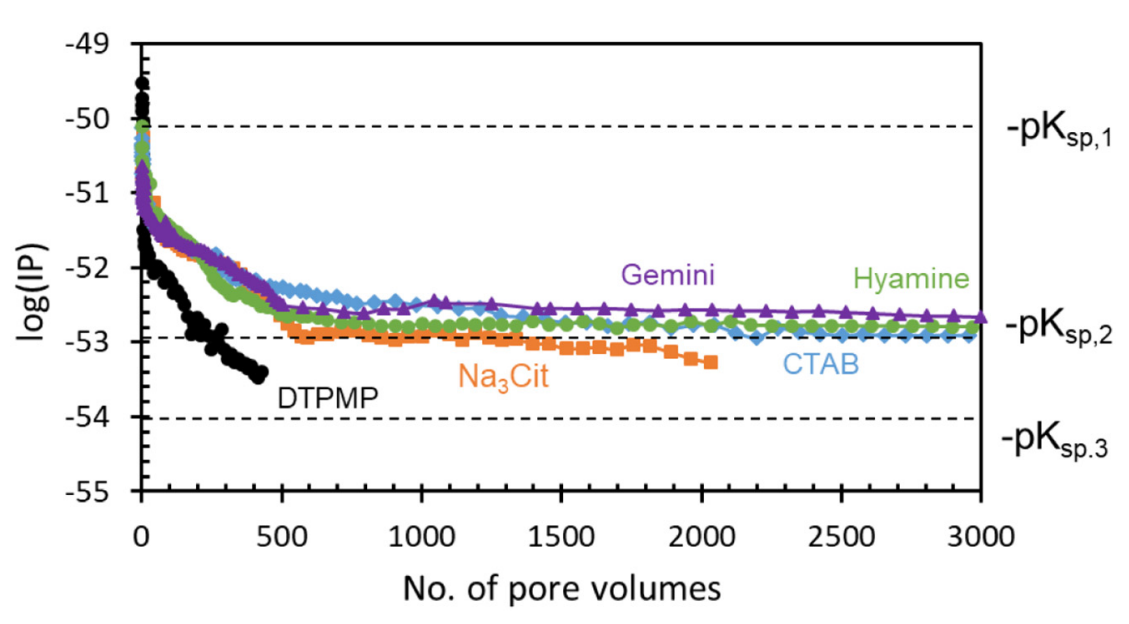

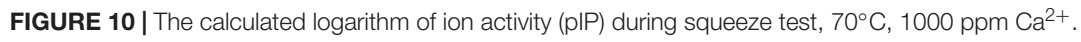

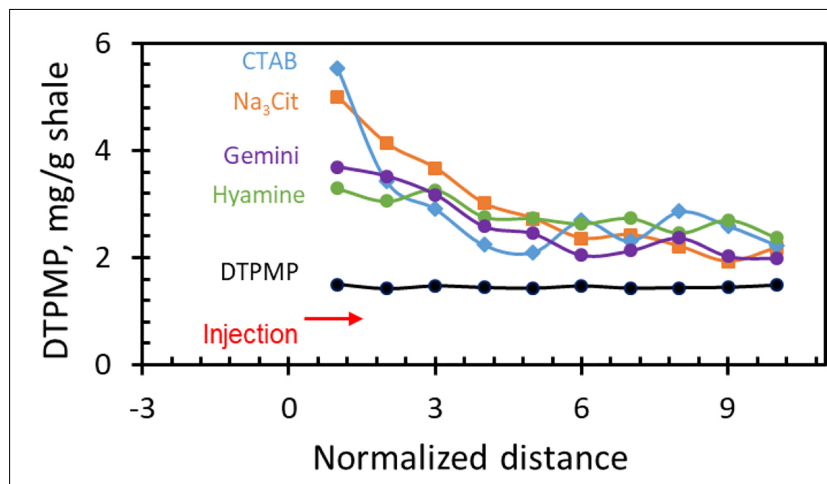

FIGURE 11 | DTPMP distribution (mg per $1 \mathrm{~g}$ shale rock) in Fayetteville shale rock after squeeze test.

$1.8\left(70^{\circ} \mathrm{C}, \mathrm{pH} 7.3\right), \mathrm{BaSO}_{4}$ scale formation with $\mathrm{SI}$ as high as 2.0 $\left(70^{\circ} \mathrm{C}, \mathrm{pH} 6.7\right)$.

To have a better understanding of the field application, normalized squeeze life (NSL), barrels of brine treated per kilogram of inhibitor added, has been introduced as (Zhang et al., 2016a):

$\operatorname{NSL}\left(\frac{\text { L of brine produced }}{k g \text { of inhibitor added }}\right)=$

$$
\frac{\text { Total effective return vol. }(\mathrm{L})}{\text { Mass of inhibitor injected }(\mathrm{mg})} \times \frac{10^{6}(\mathrm{mg})}{1(\mathrm{~kg})}
$$

According to the mass balance calculation, CTAB and Hyamine Ca-DTPMP inhibitor could maintain between 2 and 3 ppm return concentration for about 5 years, which is equivalent to $6.1 \times 10^{3} \mathrm{bbl} / \mathrm{kg}$, assuming $1 \mathrm{PV}=1$ production day, $1000 \mathrm{bbl} /$ day production rate, and a formation pore volume equivalent of $500 \mathrm{bbl}$. That for Gemini Ca-DTPMP inhibitor is 4.7 years and $5.6 \times 10^{4}$ $\mathrm{bbl} / \mathrm{kg}$ at $3 \mathrm{ppm}$ return concentration. This high return concentration may also serve as a corrosion inhibitor at the

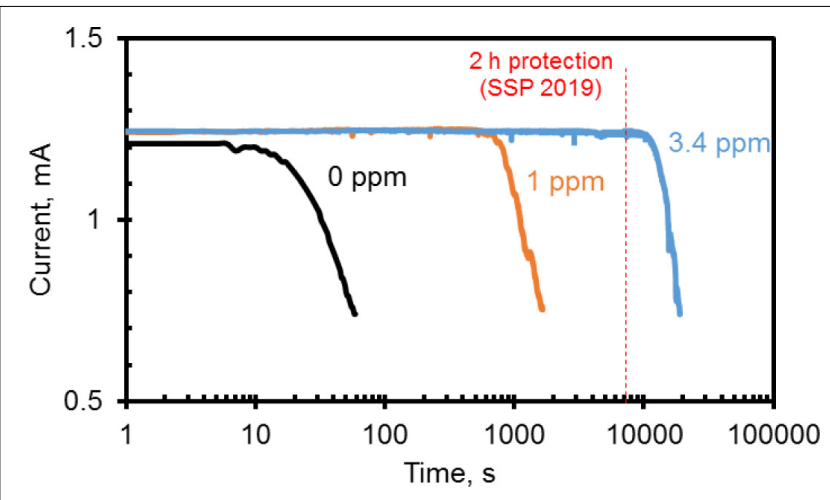

FIGURE 12 | Induction time measurement for the squeeze returned Gemini-Ca-DTPMP inhibitor, $70^{\circ} \mathrm{C}$, DTPMP, $1 \mathrm{M} \mathrm{NaCl}$, pH 5.1, $\mathrm{SI}\left(\mathrm{BaSO}_{4}\right)=1.89$

same time (Kavipriya et al., 2012). It shows a very promising potential application to the oil and gas industry. However, the aforementioned data are only based on theoretical calculation, so it needs to be carefully evaluated in the various types of brine environments.

For field application, a mechanistic squeeze prediction model, SqueezeSoftPitzer (Yan F. et al., 2014), can be used. This model has been used in several wells to accurately predict the squeeze return. If the inhibitor has been previously characterized, that data can be used to model the expected return data, but for a new inhibitor, the observed return data need to be fitted in the model first. Herein, it is assumed that each "surfactant Ca-DTPMP" should be treated as a new inhibitor that has not been previously studied in a squeeze. Figure 13 shows the comparison of Ca-DTPMP squeeze return data with the model prediction (fitting mode). The parameters of each individual inhibitor can be all well fitted. Once it is applied in another well, the squeeze return can be 


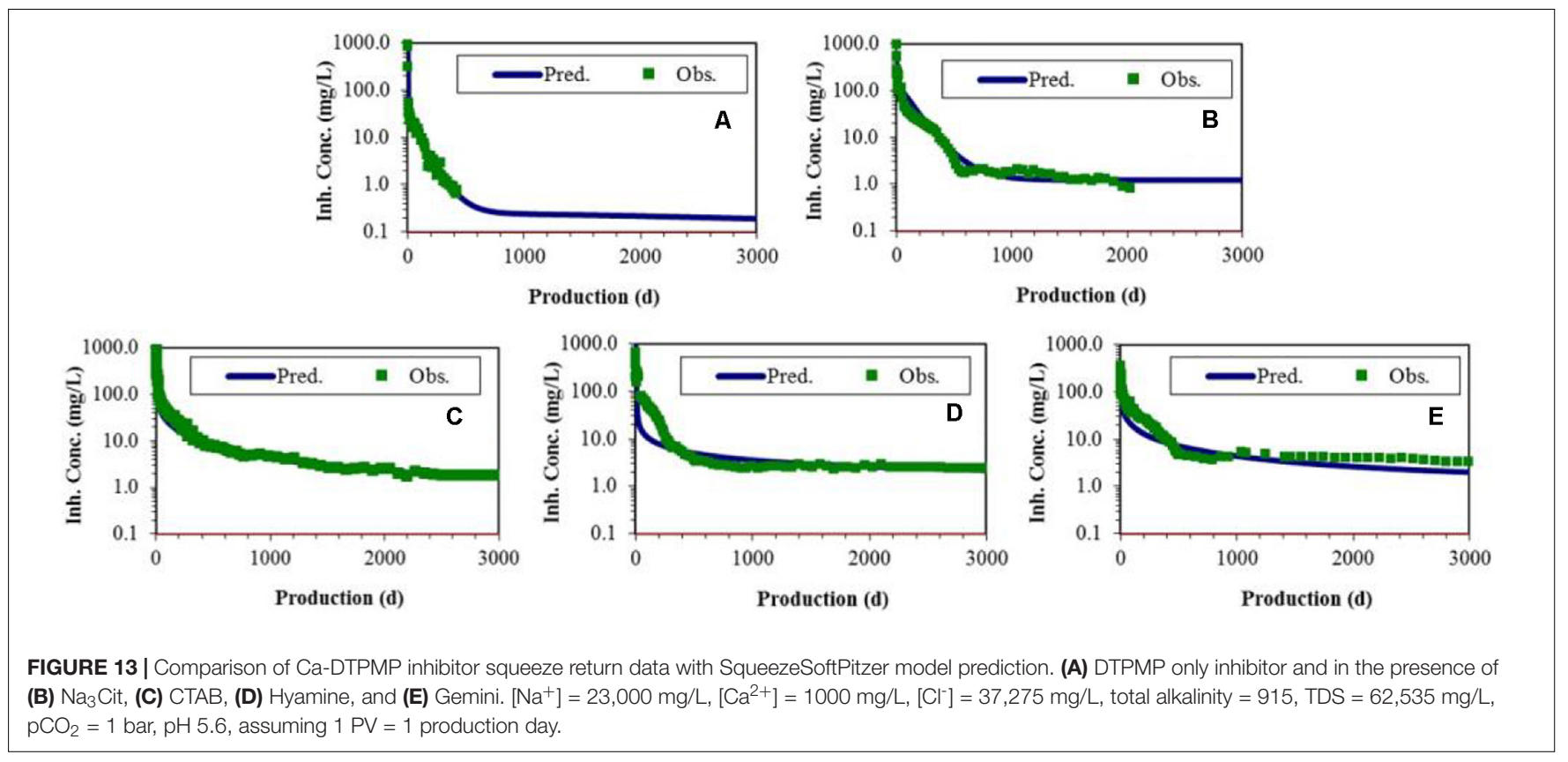

accurately predicted (prediction mode) using the previously fitted parameters.

Current work shows a promising application of Ca-DTPMP inhibitor in shale oil well fracking and production well during fracking. However, for conventional vertical and horizontal wells before fracturing, further study may be required to test the transport behavior through the core formation. In addition, the mechanism of how the surfactants can reduce particle size and how it is involved in squeeze return performance needs more detailed investigation. Further decreasing the particle size can be investigated to avoid possible pressure building up and formation damage in the field application.

\section{CONCLUSION}

High return performance was successfully achieved using CaDTPMP colloidal inhibitors prepared with different surfactants. The main conclusions that can be drawn are as follows:

- All the surfactants ( $\mathrm{Na}_{3} \mathrm{Cit}, \mathrm{CTAB}$, Hyamine, and Gemini) decreased the particle size, but Gemini was the most effective surfactant to control the Ca-DTPMP particle size to be about $\sim 500 \mathrm{~nm}$.

- The squeeze life was significantly increased using CaDTPMP nano-inhibitors. The smallest Ca-DTPMP inhibitor prepared with Gemini also shows the highest return concentration. The returned DTPMP concentration was as high as 3 ppm up to $3000 \mathrm{PV}$ tested in Fayetteville shale core. The high return performance is consistent with the adsorption and solubility of the intermediate Ca-DTPMP phase.

- Laser turbidity experiments demonstrated that the returned DTPMP is still efficient and active for preventing barite nucleation. The projected squeeze life might be as long as
5 years at $3 \mathrm{ppm}$. Therefore, the nano-Ca-DTPMP inhibitor shows promise for practical use in oil and gas production.

\section{DATA AVAILABILITY STATEMENT}

The datasets generated for this study are available on request to the corresponding author.

\section{AUTHOR CONTRIBUTIONS}

All authors listed have made a substantial, direct and intellectual contribution to the work, and approved it for publication.

\section{FUNDING}

This work was financially supported by Brine Chemistry Consortium companies of Rice University, including Aegis, Apache, BHGE, BWA, Chevron, ConocoPhillips, Coastal Chemical, EOG Resources, ExxonMobil, Flotek Industries, Halliburton, Hess, Italmatch, JACAM, Kemira, Kinder Morgan, Nalco, Oasis, Occidental Oil and Gas, Range Resources, RSI, Saudi Aramco, Schlumberger, Shell, SNF, Statoil, Suez, Total, and the NSF Nanosystems Engineering Research Center for Nanotechnology-Enabled Water Treatment (ERC1449500). The authors declare that this study received funding from the above funders. The funders were not involved in the study design, collection, analysis, interpretation of data, the writing of this article, or the decision to submit it for publication. 


\section{REFERENCES}

Dai, C., Dai, Z., Zhang, F., Zhao, Y., Deng, G., Harouaka, K., et al. (2019). “A unified experimental method and model for predicting scale inhibition," in Proceedings of the SPE International Oilfield Scale Conference and Exhibition, SPE-193586, The Woodlands, TX.

Fleming, N., Ramstad, K., Hoeth, L., and Kidd, S. (2010). "Preparation, Mechanical alteration of near wellbore mineralogy for improved squeeze performance," in Proceedings of the SPE International Conference on Oilfield Scale, SPE-130147, Aberdeen.

Franco-Aguirre, M., Zabala, R., Lopera, S., Franco, C., and Cortes, F. (2018). CaDTPMP nanoparticles-based nanofluids for the inhibition and remediation of formation damage due to $\mathrm{CaCO} 3$ scaling in tight gas-condensate reservoirs. J. Petrol. Sci. Eng. 169, 636-645. doi: 10.1016/j.petrol.2018. 06.021

Ghosh, B., and Li, X. (2013). Effect of surfactant composition on reservoir wettability and scale inhibitor squeeze lifetime in oil wet carbonate reservoir. J. Petrol. Sci. Eng. 108, 250-258. doi: 10.1016/j.petrol.2013. 04.012

Guo, X., Qiu, F., Dong, K., Zhou, X., Qi, J., Zhou, Y., et al. (2012). Preparation, characterization and scale performance of scale inhibitor copolymer modification with chitosan. J. Ind. Eng. Chem. 18, 2177-2183.

Guraieb, P., Tomson, R., and Littlehales, I. (2019). “Development of scale squeeze enhancement technology via application of metal nanoparticles coupled with polymer scale inhibitors," in Proceedings of the SPE International Conference on Oilfield Chemistry, Galveston, TX.

Horner, R., Harto, C., Jackson, R., Lowry, E., Brandt, A., Yeskoo, T., et al. (2016). Water Use and Management in the Bakken Shale Oil Play in North Dakota. Environ. Sci. Technol. 50, 3275-3282. doi: 10.1021/acs.est.5b 04079

Jordan, M., Graff, C., and Cooper, K. (2001). "Deployment of a scale squeeze enhancer and oil-soluble scale inhibitor to avoid oil production losses in low water-cut well," in Proceedings of the SPE Production \& Facilities, SPE-74330, Lafayette, LA.

Kamal, M. (2016). A review of gemini surfactants: potential application in enhanced oil recovery. J. Surfactants Deterg. 19, 223-236. doi: 10.1007/s11743015-1776-5

Kan, A., Fu, G., and Tomson, M. (2005). "Prediction of scale inhibitor squeeze and return in calcite-bearing formation," in Proceedings of the SPE International Symposium on Oilfield Chemistry, SPE-93265, The Woodlands, TX.

Kan, A., Fu, G., Tomson, M., and Al-Thubaiti, M. (2004). "A “Totally Contained" squeeze simulation approach and squeeze modeling," in NACE International, NACE-04388, New Orleans LA.

Kavipriya, K., Rajendran, S., Sathiyabama, J., and Prabha, A. (2012). A critical review of corrosion inhibition by phosphonic acids. Eur. Chem. Bull. 9, 366-374.

Kilian, L. (2016). The impact of the shale oil revolution on U.S. Oil and Gasoline Prices. Rev. Environ. Econ. Policy 10, 185-205. doi: 10.1093/reep/ rew001

Mackay, E., and Jordan, M. (2003). "SQUEEZE modelling: treatment design and case histories," in Proceedings of the SPE European Formation Damage Conference, SPE-82227, Aberdeen.

Mahmoud, M. (2014). Evaluating the damage caused by calcium sulfate scale precipitation during low- and high-salinity-water injection. J. Can. Petrol. Technol. 53, 141-150.

Poynton, N., Kelly, C., Fergusson, A., Ray, J., Webb, P., and Strong, A. (2004). "Selection and deployment of a scale inhibitor squeeze chemical for the bp miller field," in Proceedings of the International Symposium on Oilfield Scale, SPE-87466, Aberdeen.

Ruan, G., Tan, A., Yan, F., Zhang, F., Bhandari, N., Alsaiari, H., et al. (2016). “Citrate assisted metal phosphonate colloidal scale inhibitors for long-term squeeze treatments," in Proceedings of the SPE International Oilfield Scale Conference and Exhibition, SPE-179898, Aberdeen.

Selle, O., Wat, R., Vikane, O., Nasvik, H., Chen, P., Hagen, T., et al. (2003). "A Way beyond scale inhibitors-extending scale inhibitor squeeze life through bridging," in Proceedings of the International Symposium on Oilfield Scale,SPE-80377, Aberdeen.

Sutherland, L., Johnston, C., and Taylor, W. (2013). "The influence of turbulence (or Hydrodynamic Effects) on barium sulphate scale formation and inhibitor performance," in Proceedings of the SPE International Symposium on Oilfield Chemistry, SPE-164070, The Woodlands, TX.

Sutherland, L., and Jordan, M. (2016). "Enhancing scale inhibitor squeeze retention with additives," in Proceedings of the SPE International Oilfield Scale Conference and Exhibition, SPE-179888, Aberdeen.

Sydam, R., Deepa, M., and Joshi, A. (2013). A novel 1,1'-bis[4-(5,6-dimethyl-1Hbenzimidazole-1-yl)butyl]-4,4'-bipyridinium dibromide (viologen) for a high contrast electrochromic device. Organic Electron. 14, 1027-1036. doi: 10.1016/ j.orgel.2013.01.035

Takeoka, G., Dao, L., Wong, R., and Harden, L. (2005). Identification of benzalkonium chloride in commercial grapefruit seed extracts. J. Agric. Food Chem. 51, 7630-7636. doi: 10.1021/jf051 4064

Thottoli, A., and Unni, A. (2013). Effect of trisodium citrate concentration on the particle growth of ZnS nanoparticles. J. Nanostruct. Chem. 3:56.

Tomson, M., Kan, A., and Oddo, J. (1994). Acid/Base and metal complex solution chemistry of the polyphosphonate DTPMP versus temperature and ionic strength. Langmuir 10, 1442-1449. doi: 10.1021/la0001 $7 \mathrm{a} 021$

Tsagkaropoulou, G., Allen, F., Clarke, S., and Camp, P. (2019). Selfassembly and adsorption of cetyltrimethylammonium bromide and didodecyldimethylammonium bromide surfactants at the micawater interface. Soft Matter 15, 8402-8411. doi: 10.1039/c9sm01 $464 \mathrm{k}$

Vazquez, O., Mackay, E., and Sorbie, K. (2006). "Development of a non-aqueous scale inhibitor squeeze simulator," in Proceedings of the SPE International Oilfield Scale Symposium, SPE-100521, Aberdeen.

Vazquez, O., Mackay, E., and Sorbie, K. (2012). A two-phase nearwellbore simulator to model non-aqueous scale inhibitor squeeze treatments. J. Petrol. Sci. Eng. 82-83, 90-99. doi: 10.1016/j.petrol.2011. 12.030

Veisi, M., Johnson, S., Peltier, K., Liang, J., Berkland, C., Chen, M., et al. (2018). Controlled release of poly(vinyl sulfonate) scale inhibitor to extend reservoir treatment lifetime. J. Appl. Polym. Sci. 136:47225. doi: 10.1002/app. 47225

Yan, C., Kan, A., Wang, W., Yan, F., Wang, L., and Tomson, M. (2014). Sorption Study of $(-\mathrm{AlO}(\mathrm{OH})$ nanoparticle-crosslinked polymeric scale inhibitors and their improved squeeze performance in porous media. SPE J. 19, 687-694. doi: 10.2118/164086-pa

Yan, F., Kan, A., Yan, C., Wang, L., Liu, Y., and Tomson, M. (2014). "Experimental and modeling study on enhancement of squeeze treatment in sandstone by the addition of zinc ion," in Proceedings of the SPE International Oilfield Scale Conference and Exhibition, SPE-169753, Aberdeen.

Yan, F., Zhang, F., Bhandari, N., Wang, L., Dai, Z., Zhang, Z., et al. (2015). Adsorption and precipitation of scale inhibitors on shale formations. J. Petrol. Sci. Eng. 136, 32-40. doi: 10.1016/j.petrol.2015. 11.001

Zargartalebi, M., Kharrat, R., and Barati, N. (2015). Enhancement of surfactant flooding performance by the use of silica nanoparticles. Fuel 143, 21-27. doi: 10.1016/j.fuel.2014.11.040

Zhang, F., Bhandari, N., Kan, A., and Tomson, M. (2014). "Prediction of Barite Scaling Risk and Inhibition for Oil and Gas Production at High Temperature," in Proceedings of the SPE International Oilfield Scale Conference and Exhibition, SPE-169771, Aberdeen.

Zhang, P., Fan, C., Lu, H., Kan, A., and Tomson, M. (2011). Synthesis of crystallinephase silica-based calcium phosphonate nanwomaterials and their transport in carbonate and sandstone porous media. Ind. Eng. Chem. Res. 50, 1819-1830. doi: $10.1021 /$ ie $101439 x$

Zhang, P., Ruan, G., Shen, D., Kan, A., and Tomson, M. (2016a). Transport and return of an oilfield scale inhibitor reverse micelle nanofluid: impact 
of preflush and overflush. RSC Adv. 6, 66672-66681. doi: 10.1039/c6ra07 $445 f$

Zhang, P., Shen, D., Ruan, G., Kan, A., and Tomson, M. (2016b). Mechanistic understanding of calcium-phosphonate solid dissolution and scale inhibitor return behavior in oilfield reservoir: formation of middle phase. Phys. Chem. Chem. Phys. 18, 21458-21468. doi: 10.1039/c6cp03 $148 \mathrm{j}$

Zhang, P., Shen, D., Ruan, G., Kan, A., and Tomson, M. (2017). Phosphinopolycarboxylic acid modified inhibitor nanomaterial for oilfield scale control: synthesis, characterization and migration. J. Petrol. Sci. Eng. 45, 366-374. doi: 10.1016/j.jiec.2016.10.004
Conflict of Interest: The authors declare that the research was conducted in the absence of any commercial or financial relationships that could be construed as a potential conflict of interest.

Copyright (c) 2020 Gao, Dai, Wang, Zhao, Lu, Deng, Ko, Paudyal, Harouaka, Kan and Tomson. This is an open-access article distributed under the terms of the Creative Commons Attribution License (CC BY). The use, distribution or reproduction in other forums is permitted, provided the original author(s) and the copyright owner(s) are credited and that the original publication in this journal is cited, in accordance with accepted academic practice. No use, distribution or reproduction is permitted which does not comply with these terms. 